\title{
NUMERICAL ANALYSIS OF THE TEMPERATURE DEPENDENCE OF EBIC AND CL CONTRASTS
}

\author{
M. ECKSTEIN and H.-U. HABERMEIER \\ Max-Planck-Institut für Festkörperforschung, Heisenbergstrasse 1, D-7000 Stuttgart 80, Ger- \\ many
}

\begin{abstract}
Using simultaneous EBIC and CL measurements it is possible to extract the temperature dependence of the recombination efficiency from the temperature dependence of the defect contrasts, which in GaAs are dominated by the temperature dependence of the minority carrier diffusion length. Our measurements on dislocations in n-type GaAs indicate an electronic level of about $30 \mathrm{meV}$ away from a band edge related to the dislocations and give evidence for an electric field associated with the dislocation.
\end{abstract}

\section{Introduction}

In the past, especially the temperature dependence of EBIC contrasts of localized defects in Si has been used to characterize the recombination process and different recombination processes according to different defects have been proposed [1-4]. In all these models and interpretations semiconductor parameters (like the minority carrier diffusion length) have been assumed to be nearly constant [5]. The nearly temperature independent EBIC background can be taken as an experimental proof for this assumption [5]. Thus, the only quantity showing obvious temperature dependence is the recombination efficiency of the defect, causing the temperature dependence of the EBIC contrast.

In recent years first measurements of the temperature dependence of EBIC contrasts in GaAs have been published [6], but the interpretation of these measurements have turned out to be much more complicated than in Si. In an earlier paper [7] we have shown that in GaAs the temperature dependence of the bulk minority carrier diffusion length has a great influence on the temperature dependence of EBIC and CL contrasts of localized defects. Even the absolute (room temperature) value can have a dramatic effect on the shape of the contrast vs temperature profile, leading to a variety of temperature dependencies (nearly linear behaviour to transition-like behaviour), even if the recombination efficiency of the defect remains constant in the considered temperature range.

In GaAs, the strong temperature dependence of the minority carrier diffusion length brings about the strong temperature dependence of the EBIC and CL background (see Fig. 1), which show a change of at least one order of magnitude in the temperature range of 20 to $300 \mathrm{~K}$.

In this paper we demonstrate that it is possible to reconstruct the true temperature dependence of the recombination efficiency. This can be done using the temperature dependence of the diffusion length without knowing about the defect geometry. 


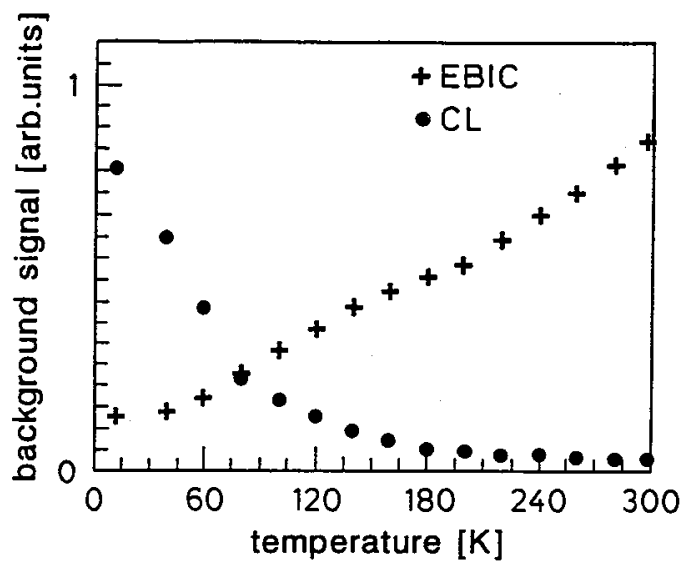

Figure 1: EBIC and CL background vs temperature for a GaAs specimen (n-GaAs:Si, $n \approx 5 \cdot 10^{17} \mathrm{~cm}^{-3}$, Al Schottky barrier). Both signals depend strongly on the temperature.

\section{Experimental Details and Results}

The specimens used for this work were cut from $<100>$ oriented GaAs wafers (n-type, $n \approx 5 \cdot 10^{17} \mathrm{~cm}^{-3}$ ). In order to measure EBIC, a $\mathrm{Cr} / \mathrm{Au}$ layer was evaporated to the rear side of the sample as an Ohmic contact, as Schottky barriers on the front side we used a $150 \AA$ thick film of Aluminium. The thickness of the Aluminium metallization is small enough to allow CL measurements of the material underneath the metallization.

Simultaneous EBIC and CL( $\left.{ }^{1}\right)$ contrast measurements were carried out at several dislocations in the temperature range from 20 to $300 \mathrm{~K}$. A detailed description of our setup can be found elsewhere [7-9]. All measurements presented in this paper were performed using a beam energy of $30 \mathrm{keV}$ and a beam current of the order of $1 \mathrm{nA}$. For the material we used in these experiments these values ensure low excitation conditions in the whole temperature range investigated [9].

A typical result of a measurement is shown in Fig. 2. Cooling down the sample, EBIC and CL contrast slightly increase, at temperatures below $150 \mathrm{~K}$ the EBIC contrast rapidly decreases and vanishes at approximately $70 \mathrm{~K}$. The CL contrast remains high down to the lowest temperatures reached.

The corresponding contrast ratio is shown in Fig. 2(b). It also shows an increase with decreasing temperature due to the decreasing minority carrier diffusion length [7].

A closer look to the EBIC contrast at low temperatures (Fig. 3) reveals that the EBIC contrast does not vanish, but goes negative for temperatures below $\approx 70 \mathrm{~K}$. This behaviour cannot be explained assuming an enhanced recombination at the defect site, it indicates that in this temperature range the reason for the contrast is different as it will be pointed out in the next paragraphs.

\section{Interpretation of Measurements}

Because there are two different effects involved in the temperature dependence of EBIC and CL contrast as it will be shown here, we divide the whole temperature range into two subranges and discuss them separately.

\subsection{Temperature Range $T>70 K$, Reconstruction of Recombination Efficiency}

In this temperature range the temperature dependence of the contrast can be completely understood by taking into account the temperature dependence of the (bulk) minority carrier diffusion length $L$. In GaAs, the diffusion length decreases with decreasing temperature. Sukegawa et al. [12] found the following

\footnotetext{
${ }^{1}$ Throughout the paper, CL stands for the integral band edge luminescence, measured with a silicon detector.
} 

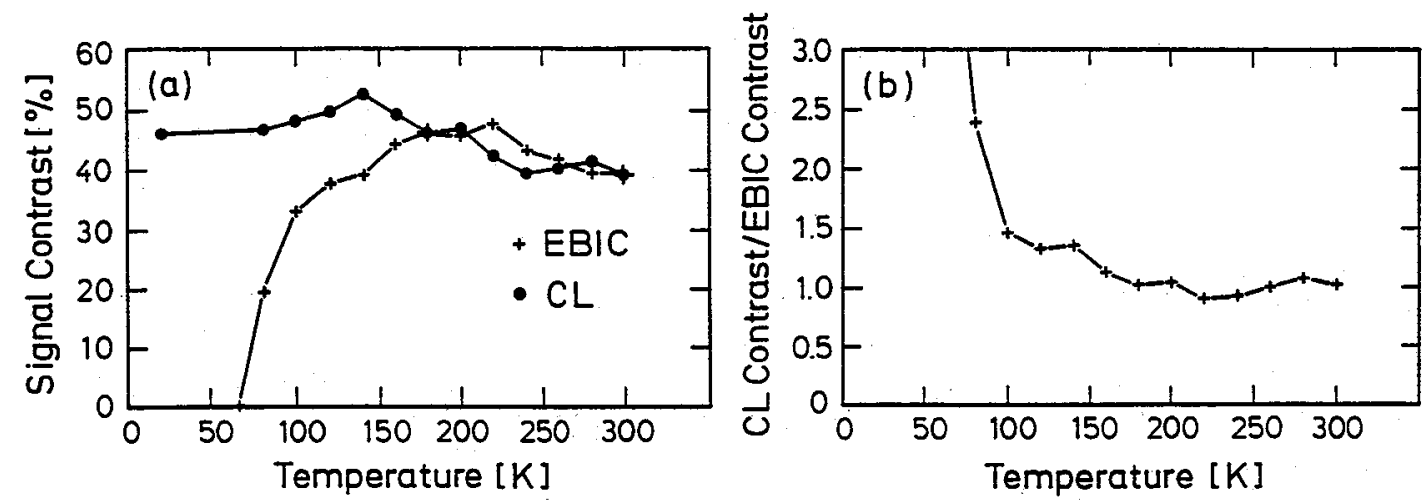

Figure 2: Quantitative measurement of the temperature dependence of EBIC and CL contrast of a grown-in dislocation in n-type GaAs (Si-doped, $n \approx 5 \cdot 10^{17} \mathrm{~cm}^{-3}$ ) in the temperature range from 20 to $300 \mathrm{~K}$.

(a) shows the temperature dependence of the contrasts,

(b) the temperature dependence of the corresponding contrast ratio.

Whereas the contrasts depend on the recombination efficiency of the defects as well as on other material parameters, the contrast ratio is independent of the recombination efficiency and only a function of geometrical and material parameters $[10,11]$.

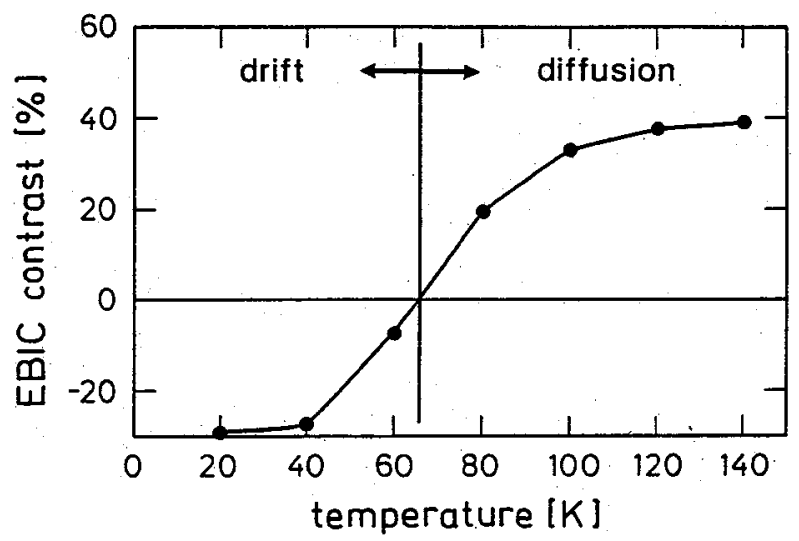

Figure 3: EBIC contrast of the same defect as of Fig. 2 for low temperatures. In the range $T<70 K$ the EBIC contrast is negative. This means that the defects are seen as bright spots. In this temperature range the contrast arises from carrier drift in an electric field (diffusion length $\approx$ zero), whereas for higher temperatures carrier diffusion is the dominating process in contrast formation. If diffusion were the only process affecting the contrast, an asymptotic behaviour of the contrast $\left(C_{E B I C} \longrightarrow 0\right)$ would be expected. 


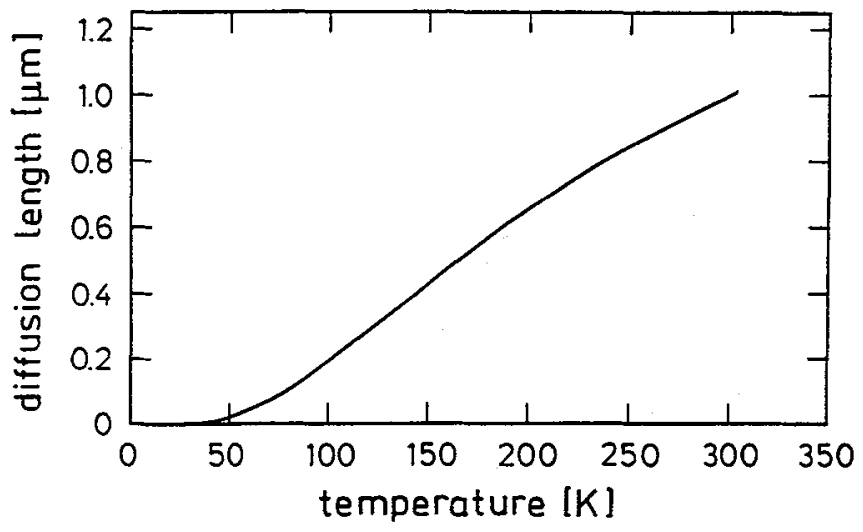

Figure 4: Temperature dependence of the diffusion length in our sample, calculated from Eq. 1 and the measured room temperature value of $1 \mu \mathrm{m}$.

temperature dependence for $L$ :

$$
[L(T)]^{2}=L_{0}^{2} \cdot \exp \left(-\frac{E}{k T}\right)
$$

with $L_{0}$ being a scaling factor and $E=43.5 \mathrm{meV}$.

Fig. 4 shows this temperature dependence for the room temperature value $L(300 K)=1 \mu m$ as measured in our samples. With this $L(T)$ a temperature dependence of the contrast ratio like the measured one (see Fig. 2(b)) can be calculated [7]. Therefore, the measured contrast vs temperature curves do not show directly the temperature dependence of the recombination efficiency but a superposition with the temperature dependence of the diffusion length.

A reconstruction of the real temperature dependence of the recombination efficiency can be done with the following procedure, using a numerical model for the simulation of EBIC and CL contrasts [9,13] based on the phenomenological theory of Jakubowicz $[10,14]$. This can be done without knowing the defect geometry.

- Taking the measured value for the contrast ratio for each temperature and the value for the diffusion length according to Eq. 1 and Fig. 4 one can calculate an "effective depth" of the $\operatorname{defect}\left({ }^{2}\right)$.

- With this "effective depth" the recombination efficiency of an equivalent defect can be calculated using a graphical method based on a set of calibration curves. It can be shown that this recombination efficiency differs from the "true" value only by a factor of the order of one [9]. The statistical error of this method can be estimated to be approx. 15 to $20 \%$.

Fig. 5 shows the reconstructed recombination efficiency, measured as an "effective capture radius" $\gamma a\left({ }^{3}\right)$. A strong increase with decreasing temperature can be seen. An Arrhenius plot (Fig. 5(b)) of these data shows a straight line with a thermal de-activation energy of about $30 \mathrm{meV}$. This can be understood assuming an energy level of about $30 \mathrm{meV}$ away from a band edge with the limiting process for the recombination being the (thermally activated) escape rate of trapped carriers.

A similar behaviour has been reported in the literature for a stacking fault in Si by Bode [1] and Kimerling [15]. Bode explains such a behaviour assuming a shallow energy level related to the stacking fault. Its recombination efficiency depends on the (temperature dependend) Fermilevel and the corresponding "demarkation energy" [16]. If the defect energy level lies between these two levels, the defect acts as a recombination site, if not, it behaves as a trap. With the Fermilevel shifting with decreasing temperature

\footnotetext{
${ }^{2}$ This has been shown in detail elsewhere [9]. This treatment can be considered as a "center-of-mass" approximation in an inhomogenious field, with the "inhomogeniety" related to the value of $L$.

${ }^{3}$ For a detailed explanation of this parameter see e.g. $[10,14]$.
} 

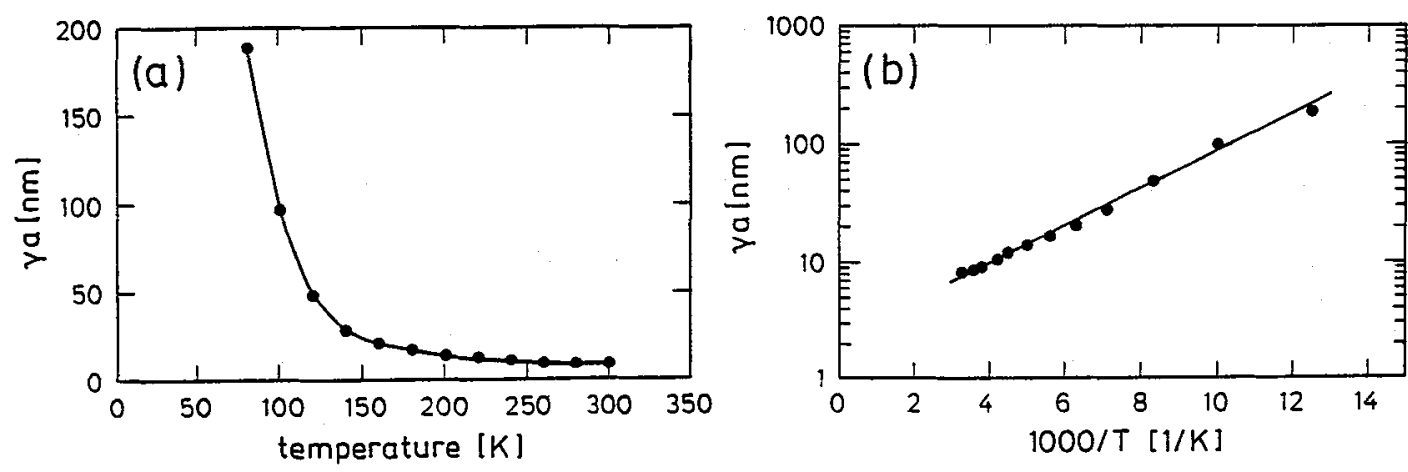

Figure 5: Reconstructed recombination efficiency, measured in terms of an "effective capture radius" (see text).
(a) linear plot
(b) Arrhenius plot, revealing a thermally deactivated process with a "deactivation energy" of about 30meV.

over a part of the gap, energy levels lying in this region become more and more effective recombination sites.

The differences between our measurement and the measurements of Bode and Kimerling (no saturation of the contrast at low temperatures; the contrast can already be observed at room temperature) are due to the high doping of our samples. This results in a very small shift of the Fermilevel with temperature $[17,18]$ leading to a very "broad" transition from trap to recombination site.

In addition, the recombination efficiency could only be detected down to approx. $80 \mathrm{~K}$, because for lower temperatures carrier drift dominates contrast formation.

\subsection{Temperature Range $T<70 K$}

Below approx. $70 \mathrm{~K}$ other effects become important for the contrast formation in this sample. This leads to a negative EBIC contrast (see Fig. 3). If the vanishing diffusion length were the only effect, an asymptotic vanishing EBIC contrast would be expected.

The fact that at these low temperatures there is still an EBIC signal (see Fig. 1) is due to the drift. of carriers in an electric field, which there is the only mechanism for carrier movement, since diffusion can be neglected (diffusion length less than a few $\mathrm{nm}$, see Fig. 4). This drift in an electric field (e.g. depletion region) then becomes important, for large values of the diffusion length (high temperature) it can be neglected.

In addition, in this sample (Si-doped) the donors begin to freeze-out at approx. $60 \mathrm{~K}$, resulting in a reduced carrier concentration. Thus, screening of potential barriers becomes less efficient and the corresponding electrical fields become weaker, but are present at a larger distance and therefore are present in a larger volume of the sample.

Thus, the fact that the defects could still be seen at these temperatures is evidence for an electric field associated with the dislocation, which influences carrier movement in the surrounding of the defect. The bright (negative) EBIC contrast can be understood assuming that the defect rejects minority carriers, leading to a higher EBIC in the vicinity of the defect. The dark CL contrast then is the result of a reduced radiative recombination at the defect due to the reduced carrier density caused by the rejection of carriers.

\section{Conclusions}

In addition to the temperature dependent recombination efficiency, two other main processes contribute to the temperature dependence of the EBIC and CL contrast of localized defects in GaAs: Diffusion, which 
dominates at high temperatures; and drift in an electric field associated with the defect at low temperatures, when diffusion is no longer possible (approx. zero diffusion length).

The reversal of the EBIC contrast in our experiment at low temperatures is direct evidence for an electric field associated with the dislocation. At this time, our experiments give no explanation of the reason for this electric field (charged states, "freeze-out" of carriers at the defect, ...). This should be subject of further investigations.

In the high temperature range, where diffusion dominates contrast formation, simultaneous EBIC and CL measurements allow reconstruction of the temperature dependence of the recombination efficiency. In our measurement, the reconstructed recombination efficiency increases strongly with decreasing temperature and reveals an energy level of $30 \mathrm{meV}$ away from a band edge associated with the dislocation.

\section{Acknowledgements}

We want to thank I. Skupin, S. Tippmann and M. Wurster for technical assistance preparing the samples. Many thanks to A. Jakubowicz for many valuable discussions.

\section{References}

[1] M. Bode: Thesis, University of Stuttgart, 1988

[2] A. Ourmazd: Cryst. Res. Tech. 16 (1981) 137

[3] A. Ourmazd, P.R. Wilshaw, G.R. Booker: J. Phys. Coll. 44 (1983) C4-289

[4] P.R. Wilshaw, G.R. Booker: Inst. Phys. Conf. Ser. 76 (1985)

[5] A. Jakubowicz, H.-U. Habermeier, A. Eisenbeiss, D. Käss: phys. stat. sol. (a) 104 (1987) 635

[6] P.R. Wilshaw, T.S. Fell, G.R. Booker: Point and Extended Defects in Semiconductors, NATO ASI SERIES B 202 (1989) 243

[7] M. Eckstein, A. Jakubowicz, M. Bode, H.-U. Habermeier: SPIE Vol. 1284 Nanostructures and Microstructure Correlation with Physical Properties of Semiconductors (1990) 228

[8] M. Bode, A. Jakubowicz, H.-U. Habermeier: Scanning 10 (1988) 169

[9] M. Eckstein: Thesis, University of Stuttgart, 1990

[10] A. Jakubowicz: J. Appl. Phys. 59 (1986) 2205

[11] L. Pasemann, W. Hergert: Ultramicroscopy 19 (1986) 15

[12] T. Sukegawa, S. Takeuchi, S. Hasegawa, H. Ueno, A. Tanaka: Bull. Res. Inst: Electron. 22/1 (1987) 47

[13] M. Eckstein, H.-U. Habermeier: to be published

[14] A. Jakubowicz: J. Appl. Phys. 57 (1985) 1194

[15] L.C. Kimerling, H.J. Leamy, J.R. Patel: Appl. Phys. Lett. 30 (1977) 217

[16] A. Rose: Concepts in Photoconductivity and Allied Problems, New York, Interscience, 1963

[17] S. Sze:Physics of Semiconductor Devices (2nd ed.), New York, Wiley, 1981

[18] W. Shockley: Electrons and Holes in Semiconductors, Toronto, D. van Nostrand Co. Inc., 1954 